\title{
Brooke Upper Extremity Rating Scale Clinical Classification
}

National Cancer Institute

\section{Source}

National Cancer Institute. Brooke Upper Extremity Rating Scale Clinical Classification. NCI

Thesaurus. Code C130304.

A standardized 6-item clinician rating scale for the assessment of upper extremity function in patients diagnosed with neuromuscular disease. The scale was developed by Brooke, et al. in 1981 to evaluate disease severity in patients with Duchenne muscular dystrophy. 